\title{
UTILIZAÇÃO DE EXTRATOS AQUOSOS DE Aspidosperma macrocarpum SOBRE DIFERENTES ESTÁGIOS DE LAGARTAS DA TRAÇA-DAS-CRUCÍFERAS.
}

\author{
Roseane Cristina Predes Trindade ${ }^{1 *}$, João Xavier de Araújo-Júnior ${ }^{2}$, Antônio Euzébio Goulart Sant'Ana ${ }^{3}$, Pedro \\ Gregório Vieira Aquino ${ }^{4}$, Ronycleide da Silva Sousa, Alds Priscila Alves de Araújo Costa
}

\begin{abstract}
${ }^{1 *}$ Centro de Ciências Agrárias da Universidade Federal de Alagoas. BR 104 N Km 85 s/n Mata do Rolo. Rio Largo - AL. CEP: 570100-000; ${ }^{2}$ Escola de Enfermagem e Farmácia, Universidade Federal de Alagoas, 57072-970 Maceió-AL;

${ }^{3}$ Instituto de Química e Biotecnologia da Universidade Federal de Alagoas. Campus A. C. Simões- Av. Lourival Melo Mota, s/n, Tabuleiro dos Martins - Maceió - AL, CEP: 57072-900;

${ }^{4}$ Laboratório de Pesquisa em Recursos Naturais, Instituto de Química e Biotecnologia, Universidade Federal de Alagoas, 57072-970 Maceió-AL;

*Autor para correspondência: Roseane Cristina Predes Trindade, roseane.predes@uol.com.br.
\end{abstract}

\begin{abstract}
RESUMO - A traça-das-crucíferas, Plutella xylostella (L., 1758) (Lepidoptera: Plutellidae), é a principal praga de brássicas em todo o mundo, principalmente por sua fácil dispersão, curto ciclo e grande capacidade de desenvolver resistência a inseticidas. Por esse motivo, a adoção de métodos de controle alternativos é importante para a elaboração de um manejo integrado para a espécie. Desta forma, avaliou-se 0 efeito de extratos aquosos de Aspidosperma macrocarpum Mart. (Apocynaceae) $(\mathrm{AM} 1$ = ramos + caule; $\mathrm{AM} 2$ = folhas e AM3 = casca do caule) em relação a diferentes fases do estágio larval de $P$. xylostella. Discos de folhas de couve (Brassica oleracea var. acephala) foram imersas em cada extrato à concentração de $1 \%(\mathrm{~m} / \mathrm{v})$ por 30 segundos, e após secos ao ar livre, foram inoculados com 12 lagartas com 1, 3 e 5 dias após a eclosão (DAE), mais a testemunha. Avaliou-se a mortalidade larval após três dias até o quinto dia. Verificou-se que em todos os tratamentos a atividade inseticida foi maior com lagarta de 1 DAE e, à medida que a lagarta se aproximou da formação da pupa (cinco DAE), essa ação inseticida não apresentou efeito. Houve diferença em relação às partes da planta, sendo a AM1 a que apresentou maior percentagem de mortalidade $(45,0 \%)$.
\end{abstract}

Palavras-chave: Plutella xylostella, planta inseticida, couve.

\section{USE OF AQUEOUS EXTRACTS OF Aspidosperma macrocarpon ON THE DIFFERENT LARVAL STAGES OF DIAMOND MOTH}

ABSTRACT - The diamondback moth, Plutella xylostella (L., 1758) (Lepidoptera: Plutellidae), is the major pest of crucifers worldwide, mainly because of its ease of dispersion, short cycle and high capacity to develop resistance to insecticides. Therefore, the adoption of alternative control methods is important for the development of an integrated management plan for the species. Therefore, the aim of this study was to evaluate the effect of aqueous extracts of Aspidosperma macrocarpum Mart. (Apocynaceae) (AM1 = branches + bark, AM2 = leaves and AM3 = wood bark) for different stages of larval stage of $P$. xylostella. Discs of leaves of cabbage (Brassica oleracea var. acephala ) were immersed in each extract at a concentration of $1 \%$ (weight / volume) for 30 seconds, and after air -dried, and inoculated with 12 caterpillars of varying days after hatching (1,3 and 5 days) plus the control, in Petri dishes. It was evaluated the larval mortality after three days of inoculation until the fifth day. It was found that in all treatment plant insecticidal activity was more pronounced during the first phase of the caterpillar (1 day after hatch) as larger percentage mortality occurred in all treatments during this period. But in stage near the pupation, five days, this action insecticide had no effect. The different parts of the plant were also differently, with the AM1 treatment showed the highest percentage of mortality with $45.00 \%$ of dead caterpillars.

Key-words: Plutella xylostella, plant insecticide, cabbage 


\section{INTRODUÇÃO}

A familia Brassicaceae abrange espécies de hortaliças de grande valor econômico, social e nutricional, tais como couve (Brassica oleracea L. var. acephala DC.), repolho (B. oleracea $L$. var. capitata $L$.), couve-flor (B. oleracea L. var. botrytis L.), brócolis (B. oleracea L. var. italica Plenck) e couve-chinesa (B. pikinensis). Além destas, existem na família várias outras espécies hortícolas e/ou oleaginosas tais como a rúcula (Eruca sativa), pak-choi (B. campestris $L$. var.chinensis Makino), couve-rabano (B. oleracea var. gongylodes), nabo-comprido (B. rapa var. rapa), rabanete (RaphanussativusL.) e mostarda-de-folha ( $B$. juncea L.) (Filgueira, 2008).

Dickson et al. (1990), relataram que o principal fator para a redução da produção das brássicas nos plantios comerciais em todas as regiões do mundo, ocorria pela frequente ação da traça-das-crucíferas, Plutella xylostella L. (Lepidoptera: Plutellidae), e atualmente, essa praga continua causando sérios prejuízos aos cultivos de brássicas pela sua ampla distribuição, como afirma Wu et al. (2012).

Essa praga causa danos graves ao limbo foliar, chegando a comprometer economicamente a cultura, ocorrendo em todas as regiões produtoras de brássicas do país apresentando picos populacionais maiores em períodos quentes e secos (Castelo Branco et al. 2001).

Devido ao uso indevido de produtos químicos e até mesmo naturais, existem vários relatos onde a $P$. xylostella apresenta resistência a esses produtos. Furlong et al. (2013) relataram uma relação de trabalhos onde $P$. xylostella apresenta resistência, e elaboraram um resumo de todos os produtos, aos quais esse inseto já apresenta resistência. Dentre esses produtos, podem-se citar: os organofosforados, carbamatos, piretroides, dos grupos químicos, a azadiractina, como ingrediente ativo, e o Bt (Bacillus thuringiensis), dentre outros.

0 gênero Aspidosperma, pertencente à família Apocynaceae, encontra-se entre as plantas de interesse medicinal, compreendendo aproximadamente 52 espécies com ampla distribuição na América do Sul, sendo bastante rica em alcaloides indólicos, dos quais muitos são utilizados em uso terapêutico. As Apocináceas produzem vários outros compostos químicos, incluindo os iridoides, glicosídeos cardioativos e alcaloides, especialmente os dos grupos indólicos complexos (derivados de triptofano e triptamina), pirrolizidínicos, esteroidais, piperidínicos, piridínicos, quinolínicos e isoquinolínicos (Henriques et al. 2001).

O gênero Aspidosperma é mais conhecido pelo uso da madeira nobre de múltipla utilização, como A. dasycarpon, A. australe, A. cylindrocarpon, A. pyricolum, A. subincanum, A. oblongum (De Paula e Alves, 1997). Outros trabalhos ressaltam essa planta como uma excelente produtora de alcaloides indólicos, de importância medicinal (Deutsch et al., 1994).

Propriedades inseticidas, especialmente contra P. xylostella (Torres et al., 2001, 2006; Trindade et al., 2008) e atividades medicinais (Deutsch et al., 1994) tem sido reportado para A. pyrifolium Mart. (Apocynaceae), uma espécie que é amplamente distribuída na caatinga do nordeste do Brasil, conhecida como pereiro-do-sertão.

A Peroba do Cerrado, A. macrocarpon Mart. (Apocynaceae) é uma planta característica de terrenos secos, decídua, seletiva xerófita, heliófita, lactescente com altura de até $18 \mathrm{~m}$, tronco com diâmetro de até $35 \mathrm{~cm}$ com ritidoma fissurado, folhas subcoriáceas, largamente elípticas a orbiculares medindo 15 a $27 \mathrm{~cm}$ de comprimento. Produz fruto folículo seco, grande achatado, lenhoso, levemente enrugado com poucas sementes (Lorenzi, 2008). Ocorre no Cerrado sentido restrito, Cerradão, e Mata Seca Semidecídua (Mendonça et al., 2008).

Sendo assim, devido à falta de trabalhos com essa espécie botânica e sabendo da quantidade de compostos químicos ativos, este trabalho teve como objetivo avaliar, em laboratório, o efeito de extratos orgânicos de A. macrocarpum (ramos e caule, folhas e casca do caule) na mortalidade larval da traça-dascrucíferas.

\section{MATERIAL E MÉTODOS}

0 presente trabalho foi conduzido no Laboratório de Entomologia: controle alternativo de pragas e em casa-de-vegetação do Centro de Ciências Agrárias da Universidade Federal de Alagoas, em Rio Largo, AL, de coordenadas geográficas $9^{\circ} 27^{\prime} 06^{\prime \prime} \mathrm{S}$ e $35^{\circ} 49^{\prime} 05^{\prime \prime}$ O, no ano de 2012.

\section{Condução da cultura}

Sementes de couve Geórgia, $B$. oleracea var. acephala, foram semeadas em casa-de-vegetação, em bandeja de isopor contendo substrato comercial 
Bioplant ${ }^{\circ}$ indicado para preparo de sementeira. Após 35 dias, as mudas foram transplantadas para local definitivo em canteiros de alvenaria preenchidos com mistura de terra preta e torta de filtro. Foram adotados tratos culturais segundo Filgueira (2008), exceto a utilização de inseticidas. Folhas de couve foram utilizadas para os experimentos a partir de 40 a 55 dias após o transplantio.

\section{Criação de Plutella xylostella}

A criação e multiplicação de $P$. xylostella foram realizadas a partir de pupas oriundas de criação do Laboratório de Entomologia Agrícola da Universidade Federal Rural de Pernambuco (UFRPE) e de uma área de plantio do Centro de Ciências Agrárias em Rio Largo - AL.

Após a emergência, os adultos eram liberados em gaiolas plásticas transparentes circulares $(12 \mathrm{~cm}$ de diâmetro $\times 15 \mathrm{~cm}$ de altura) com abertura lateral fechada com tela antiafídeo. Em cada gaiola foi colocado um pote plástico coberto com espuma umedecida, sobre 0 qual, foi colocado um disco de folha de couve medindo $8 \mathrm{~cm}$ de diâmetro para servir de substrato à postura, no sentido de simular a folha em condições de campo, e uma esponja embebida com solução açucarada a $10 \%$, na parte superior da gaiola, para alimentação dos adultos. Os discos de folhas eram substituídos diariamente, e mantidos em placas de Petri de $8 \mathrm{~cm}$ de diâmetro até a eclosão das lagartas. Lagartas recémeclodidas, oriundas de posturas realizadas em folhas de couve, foram transferidas para recipientes plásticos maiores $(20 \mathrm{~cm}$ de comprimento $\times 10 \mathrm{~cm}$ de largura $\times 5$ $\mathrm{cm}$ de altura) contendo várias folhas de couve. As folhas foram trocadas diariamente até as lagartas atingirem a fase de pupa. As pupas foram transferidas para tubos de vidro de fundo chato $(8,5 \mathrm{~cm}$ de comprimento $\times 1,5 \mathrm{~cm}$ de diâmetro), fechados com filme plástico transparente. Em cada recipiente foram realizados pequenos furos para que houvesse possibilidade de troca de ar. A cada 24 horas, após a emergência, os adultos foram transferidos para as gaiolas.

\section{Obtenção e preparo do extrato}

Os extratos orgânicos foram preparados no Laboratório de Produtos Naturais do Instituto de Química e Biotecnologia da Universidade Federal de Alagoas.
As diferentes partes de $A$. macrocarpom foram obtidas em Planaltina - GO, pelo botânico Dr. José Elias de Paula (UnB), e uma exsicata ficou depositada no herbário da UnB sob o número JEP 3767.

No preparo do extrato orgânico (etanólico) cada parte da planta, $A M 1=$ ramos + caule $(1,2 \mathrm{~kg}$ em 2L); AM2 = folhas (1,6 kg em 2L) e AM3 = casca do caule $(4,0 \mathrm{~kg} \mathrm{em} 6 \mathrm{~L})$, foram desidratados em estufa a $45^{\circ} \mathrm{C}$, moídos e submetidos à maceração em etanol $95 \%$ a temperatura ambiente $\left(27 \pm 1^{\circ} \mathrm{C}\right)$, em um percolador, em três ciclos de $72 \mathrm{~h}$ cada, sendo a solução etanólica concentrada sob pressão reduzida a $40^{\circ} \mathrm{C}$ em aparelho rotavapor, fornecendo $507,436 \mathrm{e}$ 187,1 g de extrato etanólico bruto, de AM1, AM2 eAM3, respectivamente. Após esse procedimento, 0 extrato bruto foi colocado em frasco de vidro previamente pesado e etiquetado e acondicionados aberto para a evaporação máxima do solvente.

\section{Bioensaio}

As soluções dos extratos aquosos foram preparadas com $99 \mathrm{~mL}$ de água destilada mais um grama de cada extrato bruto solubilizado com $1 \mathrm{~mL}$ de DMSO (dimetilsulfóxido), a uma concentração (peso/ volume) de $1 \%$.

Após a preparação dos extratos, discos de folhas de couve foram imersos em cada extrato por um período de 30 segundos. A testemunha foi constituída por discos imersos em água destilada com DMSO. Decorrido este tempo, os discos foram colocados sobre papel toalha e deixados ao ar livre para secagem por cerca de duas horas, e transferidos para placa de Petri contendo disco de papel de filtro umedecido com água destilada. Foram confinadas 12 lagartas de um, três e cinco dias, provenientes da criação estoque do Laboratório em cada placa de Petri. As placas foram vedadas com papel filme para evitar a fuga das lagartas.

As avaliações foram realizadas inicialmente com 3 dias, para as lagartas com 1 dia de eclosão e, diariamente, para os demais tratamentos, onde os discos de couve eram substituídos por outros sem tratamento com os extratos. Avaliou-se a percentagem de mortalidade da fase larval das diferentes fases da lagarta, através da observação diária até 0 início da fase de pupa.

0 delineamento experimental utilizado foi inteiramente casualizado com quatro tratamentos 
e cinco repetições, sendo cada uma constituída por 12 lagartas das diferentes fases. Os resultados foram submetidos à análise de variância e as médias comparadas pelo teste de Tukey usando o programa computacional ASSISTAT versão 7.6 beta (Silva e Azevedo, 2009).

\section{RESULTADOS E DISCUSSÃO}

Os dados das percentagens de mortalidade encontram-se na Tabela 1. Verificou-se que em todos os tratamentos as maiores médias de percentagem de mortalidade foram com as lagartas de 1 dia após a eclosão, ou seja, recém-eclodidas, com médias de 45,00; 30,00 e 25,00 \%, para AM1, AM2 e AM3, respectivamente.

Observou-se que à medida que as lagartas foram mudando de fase, a atividade inseticida da planta foi perdendo a capacidade, principalmente no tratamento AM1, o qual apresentou maior mortalidade com a lagarta recém-eclodida e depois a sua ação não foi tão expressiva. Os primeiros ínstares larvais, geralmente são mais suscetíveis à ação dos metabólitos secundários da planta, pelo contato direto através da ingestão do produto durante a alimentação inicial (Tabela 1).

Tabela 1. Médias \pm EP da percentagem de mortalidade das lagartas de Plutella xylostella com 1, 3 e 5 dias após a eclosão tratadas com extratos aquosos de Aspidosperma macrocarpum

\begin{tabular}{l|c|c|c}
\hline Tratamentos & Lagartas com 1 dia & Lagartas com 3 dias & Lagartas com 5 dias \\
\hline AM1 & $45,00 \pm 9,50 \mathrm{a}$ & $16,67 \pm 21,25 \mathrm{a}$ & $15,00 \pm 10,86 \mathrm{a}$ \\
\hline AM2 & $30,00 \pm 12,64 \mathrm{~b}$ & $25,00 \pm 17,68 \mathrm{a}$ & $3,33 \pm 4,56 \mathrm{~b}$ \\
\hline AM3 & $25,00 \pm 11,79 \mathrm{~b}$ & $13,33 \pm 12,64 \mathrm{a}$ & $1,67 \pm 3,72 \mathrm{~b}$ \\
\hline Testemunha & $8,33 \pm 3,72 \mathrm{c}$ & $8,33 \pm 0,00 \mathrm{a}$ & $3,33 \pm 4,56 \mathrm{~b}$ \\
\hline
\end{tabular}

Médias seguidas da mesma letra, na coluna, não diferem entre si, ao nível de $5 \%$ de probabilidade

À medida que a lagarta se aproximou da formação da pupa, com cinco dias, essa ação inseticida não apresentou efeito, com exceção do AM1 que apresentou uma média de mortalidade semelhante à lagarta com três dias.

Essa diferença de atividade em relação à fase larval do inseto também foi observado em um trabalho realizado por Roel et al. (2000) com extratos de acetato de etila de Trichilia pallida Swartz (Meliaceae) com lagartas recém eclodidas e com dez dias de idade de Spodoptera frugiperda (J.E. Smith, 1798) (Lepidoptera: Noctuidae) avaliando-se a duração e viabilidade das fases larval e pupal e a massa de pupas e concluíram que lagartas alimentadas desde a eclosão foram mais afetadas do que as alimentadas a partir dos dez dias.

E no trabalho de Roel e Vendramim (2006) no qual o extrato de acetato de etila de ramos (com folhas) de T. pallida, impregnado em folhas de milho, possui efeito residual sobre lagartas de $S$. frugiperda, mesmo com a utilização de substrato alimentar tratado sete dias antes do início do experimento. 0 extrato causou mortalidade e atraso no desenvolvimento tanto em lagartas recém-eclodidas como naquelas alimentadas a partir dos 10 dias de idade, sendo os efeitos mais pronunciados nas lagartas mais jovens.

As diferentes partes da planta também se mostraram de forma diferenciada, sendo a AM1 0 tratamento que apresentou maior percentagem de mortalidade, seguido de AM2 e AM3, com 45,00; 30,00 e $25,00 \%$, de lagartas mortas, respectivamente.

$\mathrm{Na}$ literatura existem trabalhos mostrando a atividade inseticida de espécies de Aspidosperma. Trindade et al. (2008), estudaram o extrato etanólico da madeira de A. pyrifolium $\left(5000 \mu \mathrm{g} . \mathrm{ml}^{-1}\right)$ que se mostrou promissor para o controle de $P$. xylostella com $51,7 \%$ de mortalidade. A fase hidrometanólica da partição deste extrato etanólico da madeira com acetato de etila apresentou maior eficiência com $65,0 \%$ de mortalidade larval na concentração de $4000 \mu \mathrm{g} \cdot \mathrm{ml}^{-1}$. A fração que continha a mistura de alcaloides (aspidofractinine, 15-demethoxypyrifoline e $\mathrm{N}$-formylaspidofractinine) apresentou uma eficiência de mortalidade de $100 \%$ na concentração de $4000 \mu$ g $\mathrm{ml}^{-1}$.

Como também, Medeiros (2007) estudou a ação de $A$. pyrifolium como ação larvicida eficiente, com $100 \%$ de mortalidade em apenas 24 horas da aplicação do extrato da casca e raiz contra o mosquito da dengue Aedes aegpty (Diptera: Culicidae), mesmo quando 
utilizada em concentrações inferiores à concentração padrão de $200 \mathrm{~g} / \mathrm{L}$.

0 extrato etanólico da folha de pereiro-dosertão, nas concentrações de $50 \mathrm{mg} / \mathrm{L}$ e $10 \mathrm{mg} / \mathrm{L}$, apresenta efeito insetistático sobre Heliothis virescens (Fabricius, 1781) (Lepidoptera: Noctuidae), evidenciado pela diminuição na fecundidade e no número de fêmeas/população (razão sexual) (Silva et al. 2008).

Existe também um estudo com ação do pereiro para o controle de carrapatos, onde Santos (2010), estudou a ação do extrato vegetal de folhas de $A$. piryfolium com atividade antiprasitária de $91 \%$, contra o carrapato do boi Rhipicephalus (Boophilus) microplus (Canestrini, 1887).

Torres et al. (2001) avaliaram diferentes partes (folhas, ramos, casca, frutos e raízes) na concentração de $10 \%$ de 13 espécies vegetais no controle de $P$. xylostella e relataram que nove tratamentos diferiram da testemunha. Dentre esses tratamentos, a $A$. pyrifolium, $A$. indica e uma formulação a base de nim apresentaram mortalidade de $100 \%$ da fase larval.

Como também, Torres et al. (2006) verificaram efeitos tóxicos de extratos aquosos de amêndoas de $A$. indica, córtex do lenho de $A$. pyrifolium e frutos de Melia azedarach (Meliaceae) com uma concentração letal de 0,6; 7,0 e 12,5\% para a $P$. xylostella.

Com aplicação dos extratos aquosos de $A$. macrocarpum sobre lagartas de $P$. xylostella, é possível concluir que os primeiros ínstares são mais suscetíveis à ação dos extratos e que o extrato dos ramos e caule (AM1) de A. macrocarpum apresenta melhor atividade inseticida na concentração de $1 \%$.

\section{REFERÊNCIAS BIBLIOGRÁFICAS}

CASTELO BRANCO, M.; FRANÇA, F.H.; MEDEIROS, M.A.; LEAL, J.G.T. Uso de inseticidas para o controle da traça-do-tomateiro e traça-das-crucíferas: um estudo de caso. Horticultura Brasileira, 2001, 19, 1, 60-63.

DE PAULA, J.E.; ALVES, J.L.H. Madeiras nativas. Anatomia, dendrologia, dendrometria, produção e uso. Brasília - DF, 1997. 543p.

DEUTSCH, H.F.; EVERSON, M.A.; DRESCHER, P.; SPARWASSER, C.; MOOSEN, P.O. Isolation and biological-activity of aspidospermine and quebrachamine from an Aspidosperma tree source.
Journal of Pharmaceutical and Biomedical Analysis, $1994,12,1283-1287$.

DICKSON, M.H.; SHELTON, A.M.; EIGENBRODE, S.D.; VAMOSY, M.L.; MORA, M. Selection for resistance to diamondback moth (Plutella xylostella) in cabbage. Hortscience, 1990, 25, 1643-1646.

FILGUEIRA, F.A.R. Novo manual de olericultura: agrotecnologia moderna na produção e comercialização de hortaliças. 3. ed. Viçosa. MG: Universidade Federal de Viçosa, 2008, 421p.

FURLONG, M.J.; WRIGHT, D.J.; DOSDALL, L.M. Diamondback moth ecology and management: problems, progress, and prospects. Annual Reviews Entomology, 2013, 58, 517-541.

HENRIQUES, A.T.; KERBER, V.A.; MORENO, P.R.H. Alcalóides: generalidades e aspectos básicos. Cap. 29, p. 651-666. In: SIMÕES, C.M.O.; SEBENKEL, E.P.; GOSMANN, G.; MELLO, J.C.P.; MENTZ, L.A.; PETROVICK, P.R. Farmacognosia da planta ao medicamento. 3ed. Rev. Porto Alegre / Florianópolis: Ed. Universidade / UFRGS / Ed. Da UFSG / 2001. 833p.

LORENZI, H. Árvores brasileiras: manual de identificação e cultivo de plantas arbóreas nativas do Brasil. Vol. 1. Nova Odessa, SP: Instituto Plantarum, 2008.

MEDEIROS, V.F. Potencial larvicida de extratos de plantas regionais no controle de larvas de Aedes aegypti (Diptera: Culicidae). Dissertação de Mestrado Universidade Federal do Rio Grande do Norte. Centro de Biociências. Programa de Pós-graduação em Ciências Biológicas. 2007. 69f.

MENDONÇA, R.C.; FELFILI, J.M.; WALTER, B.M.T.; SILVA JÚNIOR, M.C.; REZENDE, A.B.; FIGUEIRAS, T.S.; NOGUEIRA, P.E.; FAGG, C.W. Flora Vascular do Bioma Cerrado: Checklist com 12.356 espécies. In: Sano, S. M.; Almeida, S. P.; Ribeiro, J. F. (ed.). Cerrado: Ecologia e Flora Vol. 2. $1^{\text {a }}$ ed. Brasília, DF: Embrapa Informação Tecnológica, 1279p, 2008.

ROEL, A.R.; VENDRAMIM, J.D.; FRIGUETTO, R.T.S.; FRIGUETTO, N. Efeito do extrato acetato de etila de Trichillia pallida Swartz (Meliaceae) no desenvolvimento e sobrevivência da lagarta-do-cartucho. Bragantia, 2000, 59, 1, 53-58. 
ROEL, A.R.; VENDRAMIM, J.D. Efeito residual do extrato acetato de etila de Trichilia pallida Swartz (Meliaceae) para lagartas de diferentes idades de Spodoptera frugiperda (J. E. Smith, 1797) (Lepidoptera: Noctuidae). Ciência Rural, 2006, 36,4, 1049-1054.

SANTOS, P.B. Contribuição ao estudo químico, bromatológico e atividade biológica de angico Anadenanthera colubrina (Vell.) Brenan. Var. cebil (Gris.) Alts e pereiro Aspidosperma pyrifolium Mart. Patos - PB. Programa de Pós-graduação em Zootecnia. Dissertação de Mestrado. 2010. $46 f$.

SILVA, D.F.S.; OLIVEIRA, Y.A.; CORREIA, A.A.; WANDERLEY-TEIXEIRA， V.; TEIXEIRA， A.A.C.; ALVES, L.C.; CÂMARA, C.A.G. Efeito do extrato etanólico da folha do pereiro-do-sertão (Aspidosperma pyrifolium Mart.) sobre parâmetros biológicos de Heliothis virescens (Fabricius, 1781) (Lepidoptera: Noctuidae). Biológico, 2008, 70, 2, 107-216.

SILVA, F.A.Z.; AZEVEDO, C.A.V. Principal Components Analysis in the Software Assistat-Statistical Attendance. In:WORLD CONGRESS ON COMPUTERS IN AGRICULTURE, 7, Reno-NV-USA: American Society of Agricultural and Biological Engineers. 2009.
TORRES, A.L.; BOIÇA JUNIOR, A.L.; MEDEIROS, C.A.M.; BARROS, R. Efeito de extratos aquosos de Azadirachta indica, Melia azedarach e Aspidosperma pryrifolium no desenvolvimento e oviposição de Plutella xylostella. Bragantia, 2006, 65, 3, 447-457.

TORRES, A.L.; BARROS, R.; OLIVEIRA, J.V. Efeito de extratos aquosos de plantas no desenvolvimento de Plutella xylostella (L.) (Lepdoptera: Plutellidae). Neotropical Entomology, 2001, 30, 151-156.

TRINDADE, R.C.P.; SILVA, P.P.; ARAÚJO-JÚNIOR, J.X.; LIMA, I.S.; DE PAULA, J.E.; SANT'ANA, A.E.G. Mortality of Plutella xylostella larvae treated with Aspidosperma pyrifolium ethanol extracts. Pesquisa Agropecuária Brasileira, 2008, 43: 1813-1816.

WU, Q.J.; ZHANG, S.; YAO, J.; XU, B.; WANG, S.; ZHANG, Y. Management of diamondback moth, Plutella xylostella(Lepidoptera: Plutellidae) by mating disruption. Insect Science, 2012, 19: 643-648. 